\title{
A Hybrid Off/On-Lattice Model of Emergence and Maintenance Autopoiesis
}

\author{
Olivier Wang ${ }^{1,2} *$ René Doursat $^{1,3}$ and Paul Bourgine ${ }^{1,3}$ \\ ${ }^{1}$ Ecole Polytechnique, Paris, France - European Erasmus Mundus Master's in Complex Systems Science (MCSS) \\ ${ }^{2}$ IBM France Lab, Paris, France \\ ${ }^{3}$ Complex Systems Institute, Paris Ile-de-France (ISC-PIF), CNRS UPS3611, Paris, France \\ *corresponding author: olivier.wang@polytechnique.edu
}

\begin{abstract}
We propose an original 2D agent-based model of biological "autopoiesis", the process by which a cell creates and continuously regenerates itself, considered one of the defining characteristics of life. In the space of our simulations, the positions of free molecules are continuous, and polymerized membrane components are regularly arranged (plus noise). While autopoiesis commonly refers to the self-driven maintenance of a system, we also follow Varela's historical study of emergence, and show that the same model can account for both selfperpetuation and self-formation - a step toward uniting the three main perspectives on life: origins, autopoiesis, and replication. Exploring different initial and environmental conditions, we observe that destructive reactions are important for the survival of our autopoietic system, and evaluate their impact on its lifespan. The tendency of cells to form spurious outgrowths is counteracted by moderate decay of the membrane.
\end{abstract}

\section{Introduction}

The study of life and consciousness construed as high-level, abstract systemic properties, beyond their biochemical composition, has led to the concept of "autopoiesis", a term coined by Maturana and Varela (1973) to refer to the self-creation and self-repair abilities of organisms. The initial idea of defining living systems as fundamentally autopoietic is well accepted today, and has also been broadened to include cognitive systems (Bourgine and Stewart, 2004). Yet, autopoiesis is still at the center of a long-lasting debate (Fleischaker, 1992), where critics object to the overly theoretical nature of the concept and deem self-referentiality without external references meaningless (Swenson, 1992). By contrast, other fields such as sociology (Luhman, 1986) have embraced autopoietic thinking and imported it into their research. The original 1973 definition stated that:

An autopoietic system is a machine organized (defined as a unity) as a network of processes of production (transformation and destruction) of components that produces the components which: (i) through their interactions and transformations continuously regenerate and realize the network of processes (relations) that produce them; and (ii) constitute it (the machine) as a concrete unity in the space in which they exist by specifying the topological domain of its realization as such a network.

Many variants have been proposed since. We base our model on Bourgine and Stewart's more recent approach (2004):
An autopoietic system is a network of processes that produces the components that reproduce the network, and that also regulates (from inside) the membrane conditions necessary for its ongoing existence as a network.

We will also include the less explicited notion that, since the components are what reproduces the network, the processes cannot be based on global variables or global properties of the system, but must rely on purely local interactions.

These different viewpoints on autopoiesis have produced various computer models. Agent-based simulations, where a large number of discrete units are updated sequentially or synchronously, have been the tool of choice to illustrate the concept of autopoiesis since its inception. The first attempt (Varela et al., 1974) used cellular automata (CA), and was pursued and extended by Zeleny (1977) who formalized the computational model and explored other scenarios, in particular ones involving more catalysts in the environment. A later analysis of this original model by McMullin and Varela (1997) pointed out that, although it was missing a critical "additional interaction", the algorithm did achieve autopoiesis when membrane creation was inhibited in its interior neighborhood. Further developments have also allowed movement of the simulated membrane (Breyer et al., 1998; McMullin and Groß, 2001).

The majority of other simulated autopoietic systems have been confined to a CA grid, such as Beer's analysis of a glider in the Game of Life (Beer, 2004), or the 3D lattice artificial chemistry of Ono and Ikegami $(1999,2000,2001)$ based on hexagonal units, as was Sirmai's 2D "morphautomaton" (2011, 2013). Beer proved that there can be other types of autopoietic systems than molecular ones imitating the biochemistry of life. He also emphasized that proper definitions of the system and its boundary are essential when determining whether a system is autopoietic or not. Ono and Ikegami showed that particle interaction models can exhibit autopoietic behavior, and hypothesized about the structure of early life forms on Earth. Sirmai illustrated how the self-maintenance mechanisms of an autopoietic system could also give rise to self-replication when adding a few interactions.

The relative lack of autopoietic simulations in continuous space can be partially explained by the computational cost compared to discrete models. Whereas, more generally, the field of artificial chemistry has produced continuous implementations (Hutton, 2007; Ono and Ikegami, 2001: Chap. 3) these have not addressed autopoiesis per se. Moreover, the 
continuous case is only described qualitatively, while quantitative results are generally produced by a discrete approximation on a lattice. A review of computational models of autopoiesis was written by McMullin on the $30^{\text {th }}$ anniversary of the concept's discovery (McMullin, 2004).

In this paper, the molecular dynamics is an artificial chemistry that happens for the most part in continuous 2D space. A discrete lattice component is still present to constrain the locations and behavior of membrane particles, once they have bound to each other. We take inspiration from Bourgine and Stewart (2004) to define the reactions, and simplify the system so that it involves fewer classes of components. Two possible outcomes are analyzed, one corresponding to the usual sense of autopoiesis as self-maintenance, and the other representing the ability of the system to create itself from a single element, or emergence, which is closer to the etymology of the term. The latter is necessary to the former and to self-reproduction.

\section{A Model of Cell Autopoiesis}

\section{Particles, Membrane, and Environment}

We consider a continuous $2 \mathrm{D}$ environment, the world. It is filled with particles animated by Brownian motion (i.e. which follow straight trajectories between two collisions), called substrate particles and denoted by $S$ (pink disks in Figs. 1-4). Their density, i.e. average number per lattice square, is set to 0.4 . In the world, we observe the creation and/or survival of an autopoietic system, a cell, made of two other types of particles, monomers $M$ (yellow or green disks) and component particles $C$ (blue disks). The cell is characterized by a semipermeable membrane arising from monomers binding together. Monomers have a diameter of 1 and are locked into lattice positions regularly spaced by the same unit, with a small Gaussian vibration of width $\varepsilon$. The membrane serves as a boundary for the system and can self-repair as a whole. Monomers randomly decay into waste particles $W$ (orange disks), at a low rate $\delta$, thus gradually damaging the membrane over time. Counteracting this, the membrane is also repaired by component particles that are present in the enclosed space and transform into monomers. Details are explained below.

The monomers of the membrane are permeable to the small substrate particles; they do not impede their movement. On the other hand, the substrate, component and waste particles all collide and interact with one another in a way similar to gas molecules. Monomers have an orientation vector (Fig. 2a) to mark the inside and the outside of the membrane, i.e. the two half-spaces created by a dividing line orthogonal to that orientation and running through the center of the molecule. We also say that the inside is "behind" the monomer, and the outside is in the "front". The local curvature of the membrane is a fixed property of the monomers, arising from their internal molecular structure. It is modeled here by a constant angle $\alpha$ between two neighboring orientation vectors.

Upon colliding, these various particles react in different ways depending on their type. We consider in this model three key interactions resulting in the creation or destruction of particles (Fig. 2): the synthesis of components, the repair of membrane monomers, and the decay of monomers.

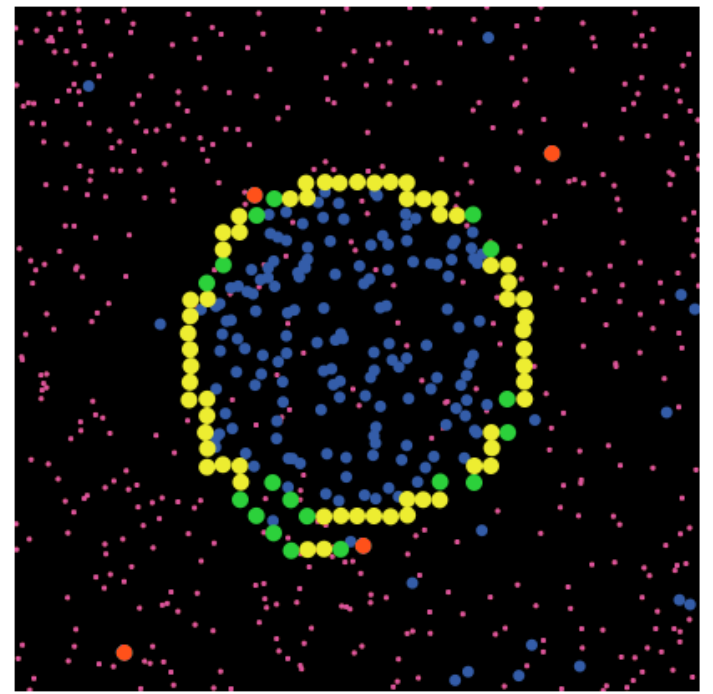

Figure 1: Example of autopoietic cell. Pink: substrate particles $S$; blue: components $C$; yellow: doubly bonded monomers, $M_{2}$; green: simply bonded, $M_{1}$, or unbonded monomers, $M_{0}$; orange: waste particles, $W$. In the top-left quadrant of the membrane, two pairs of neighboring $M_{1}$ 's exhibit holes of size $\sqrt{2}-(2 \times 0.5)=0.41 \pm 2 \varepsilon$. Near the upper hole, one $M_{2}$ has just decayed into a $W$. In the top-right quadrant, there is a larger hole of size $\sqrt{ } 5-1=1.24 \pm 2 \varepsilon$. At the bottom-left, the system has formed two layers, starting a spiral shape. Thus it is not autopoietic because its membrane is not closed. Time tick: $t_{i}=350$. Parameters: membrane curvature angle $\alpha=8^{\circ}, M$-decay rate $\delta=0.05 \%, C$-saturation level $c=2$ and radius $r=10, M$-vibration width $\varepsilon=0.05$.

Synthesis: $\boldsymbol{S}+\boldsymbol{S} \stackrel{\boldsymbol{M}}{\rightarrow} \boldsymbol{C}$ Two substrate particles can produce one component particle under specific conditions: the reaction must be catalyzed by a nearby monomer $M$ and only if it happens "behind" it (Fig. 2a). Moreover, it should not be inhibited by too many other $C$ 's in the local environment: the density of $C$ particles in a given radius $r$ should stay below a saturation level $c$. If these conditions are met, then the two $S$ particles disappear and a $C$ is put in their place, with averaged speed and direction. These two conditions are local versions of Bourgine and Stewart's model (2004).

Repair: $\boldsymbol{C}+\boldsymbol{M}_{\mathbf{1}} \rightarrow \boldsymbol{M}+\boldsymbol{M}_{2}$ This reaction transforms a freely moving component into a monomer that integrates the chain of monomers forming the membrane. This can involve either filling a hole that has appeared in an existing membrane ("maintenance autopoiesis"), or building a new membrane ("emergence autopoiesis"). Thus, by "repair", we also mean the series of reactions that make a single $M$-seed grow into a complete autopoietic system. Like "synthesis", it also requires certain local conditions to be fulfilled: $C$ particles transform into $M$ only when they are close to a membrane hole or extremity, i.e. a "simply bonded" $M$, a monomer with only one neighbor, denoted by $M_{1}$ (in green). In addition, $C$ must also be located within a specific arc of space (a 2D cone) in relation to the neighboring $M$, which we call receptor arc (Fig. 2b). It is defined as the domain "behind" $M$ where the angle between the space-dividing line and the $M C$ segment is smaller than the curvature angle $\alpha$. 


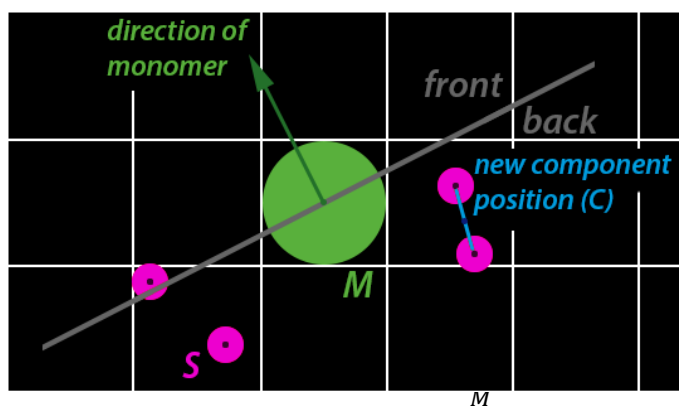

(a) Synthesis: $S+S \stackrel{M}{\rightarrow} C$

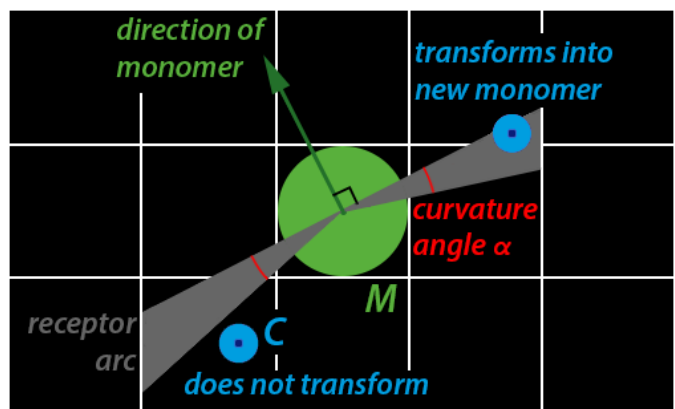

(b) Repair: $C+M_{1} \rightarrow M+M_{2}$
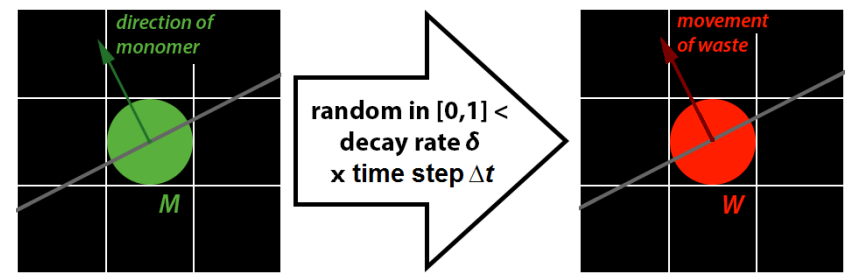

(c) Decay: $M \rightarrow W$

Figure 2: The three fundamental reactions of the model. (a) "Synthesis": two $S$ particles (pink) located in the same square can create a $C$ (blue) if an $M$ (green) is present in a neighboring square and if they are "behind" it (verified on the right, not on the left). (b) "Repair": only a $C$ located in a square adjacent to an $M$ and inside the receptor arc (gray area) may transform into a new $M$, added to the membrane. (c) "Decay": with low probability at each iteration, any $M$ can become a waste particle $W$, which receives an initial speed in the direction of $M$ 's vector (and norm 10 here).

Decay: $\boldsymbol{M} \rightarrow \boldsymbol{W}$ This is the simplest reaction, as it involves a single monomer randomly becoming waste (Fig. 2c). The probability of decay per time unit, $\delta$, is constant and uniform over all $M^{\prime} \mathrm{s}$; it is independent from the age of the $M$ particle.

\section{Physics simulation}

Our simulated environment does not use a physics engine, unlike other artificial chemistry works, but was more simply implemented in the NetLogo platform (Wilensky, 1999). Although the disk-shaped particles have floating number coordinates, their collisions are not modeled by solid bodies. Instead, we repurposed a "dynamic billiard" gas model that partitions the world into square domains where particles interact. To ensure the detection of collisions, the duration of one time step was dynamically adjusted so that no particle could
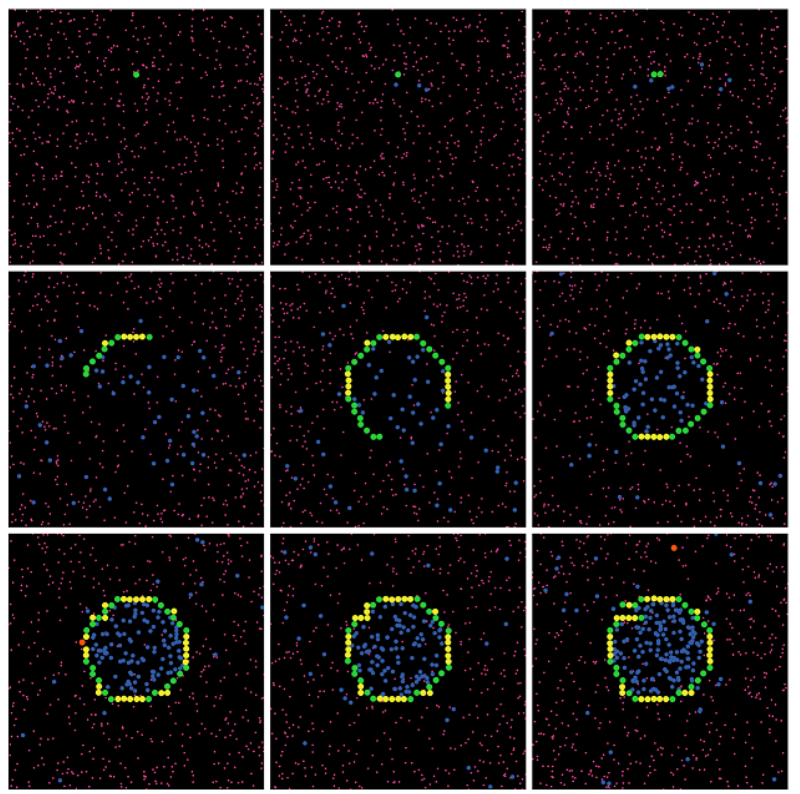

Figure 3: Example of "emergence autopoiesis" followed by temporary "maintenance autopoiesis". Top: At first, a few components $C$ are created by $S$ particles colliding in the vicinity of the seed monomer $M$. Then, the $C$ 's start building a circular membrane by transforming into new $M$ 's (ticks $t=0,0.27,1$ ). Emerging radius is 8 (from an angle $\alpha=8^{\circ}$ ). Middle: The chain grows and closes itself (ticks $t=64,126,151)$. Bottom: For a while, the cell is autopoietic, as holes appearing from the decay of $M$ 's are filled with new ones (ticks $t=210.5,240$ ). Eventually, however, the membrane starts spiraling and the cell dies (tick $t=364.4)$. Parameters identical to Fig. 1. (A video can be seen at http://tinyurl.com/locy7qy.)

travel farther than one square at every iteration. Adding these variable time steps yields time ticks, denoted by $t_{i}$, where $i$ is the iteration index. The $i$-th time step is $\Delta t_{i}=t_{i+1}-t_{i}$, and the total duration of a run is $t_{N}$, where $N$ is the last iteration (typically, $t_{N}=3000$ in this study, which corresponds to an average $N \approx 10000$ ). A similar model is available online (Pelaez, 2009), although it does not demonstrate an actual autopoietic system since it constrains the membrane to a preassigned location and allows its formation only there.

In this environment, we also limit the degrees of freedom of the membrane. Bonded $M$ particles cannot move away from the integer coordinates they are pegged to, only vibrate around them. At every time step, a small random vector is added to the center location (see Rule 9 below). The membrane as a whole cannot deform or drift. Note that an $M$ is only labeled "unbonded" $\left(M_{0}\right)$, "simply" $\left(M_{1}\right)$, or "doubly bonded" $\left(M_{2}\right)$ by proximity to other $M$ 's, but physically it behaves the same.

\section{Algorithm}

In sum, each iteration $i$ with a time step $\Delta t_{i}$ essentially consists of the following rules and actions:

1. "Displacement" rule: recalculate the velocity vector $\vec{v}$ of the $C$ and $W$ particles that bounce off an $M$. Update the positions of all moving particles by adding $\vec{v} \Delta t_{i}$. Particles that exit the world disappear permanently. 
2. "Replenish" rule: create new $S$ particles at the edge of the world to simulate a solution of constant $S$-concentration.

3. "Repair" reaction rule: test pairs of neighboring $C$ 's and replace them with new $M$ 's where appropriate (see above).

4. "Synthesis" reaction rule: change the properties of colliding $S$ particles: either create one $C$ if proper conditions are met (see above), or modify their $\vec{v}$ if they only rebound.

5. "Decay" reaction rule: for each $M$, if a uniformly random number in $[0,1]$ is less than $\delta \Delta t_{i}$, replace it with a $W$.

6. Increment the iteration counter: $i \rightarrow i+1$.

7. "Time update" rule: determine the length of the next time step $\Delta t_{i+1}$ by polling the velocities $\vec{v}$ of all the moving particles and making sure $\left\|\vec{v} \Delta t_{i+1}\right\|$ does not exceed 1 .

8. "Vibration" rule: every full unit of time, add a small random vector, with uniform angle in $[0,2 \pi]$ and Gaussian norm of width $\varepsilon$, to each $M$ around its lattice position.

This algorithm is used for both types of initial condition (existing membrane and single monomer) and runs until tick $t_{N}=3000$ for all the results presented in this article.

\section{System definition, exploration, and measure}

Through this model, we want to study the autopoietic properties of a class of systems defined by a closed, roughly circular membrane made of bonded particles of one type (here, monomers $M$ ) and enclosing freely moving particles of another type (here, components $C$ ). The topology of a system can be assessed by the distribution of holes in the chain of monomers composing the membrane, including their number, locations and sizes. The size $h$ of a hole is defined as the shortest distance between two neighboring $M_{1}$ 's (simply bonded monomers; green disks in figures), i.e. the diagonal distance between their centers minus 1 (twice their radius). Given a maximum hole size $\lambda$ (in general 1.5 , just above $\|(2,1)\|-1$ ), we calculated the number of holes $n_{\lambda}$ bigger than $\lambda$ and said that

- the membrane was "closed" if $n_{\lambda}=0$,

- the membrane was "viable" if $n_{\lambda}=1$,

- the membrane was "broken" if $n_{\lambda} \geq 2$.

In the last case, the presence of two holes or more created at least two disconnected pieces in the 2D world. (Naturally, these definitions would need to be modified in $3 \mathrm{D}$, replacing punctual holes with lines or closed paths on the surface of the membrane.) Note that, since we only considered holes larger than 1.5, a closed or viable membrane could still contain several smaller holes (typically of size 0.4 or 1 , rarely 1.24 ), which were called "pores". This is a fundamental characteristic of real-world cells and other autopoietic systems: while presenting a well-formed and stable membrane structure, they also kept a partial "openness" to the environment, letting material flow inward and outward through small channels.

In addition, we verified that the membrane remained roughly circular, i.e. did not form a spiral as in Figs. 3-4 or an open thread. These unwanted structures did not necessarily contain regular holes - or they could be said to contain $M_{1}$ 's on the edge of "infinite" holes. For this, we checked that the virtual spokes connecting each $M$ to the center of the world were not passing through any other $M$ particle.
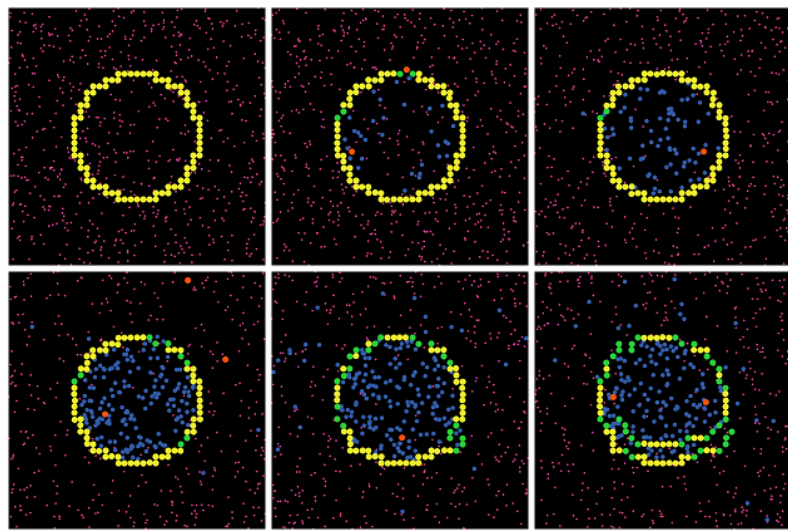

Figure 4: Example of temporary "maintenance autopoiesis". The system is initialized with a circular membrane of radius 10 (composed of $80 \mathrm{M}$ 's). Small holes are repaired in the begining (top: ticks $t=0,1,6)$, more holes appear later (bottom: tick $t=46$ ) and the membrane eventually breaks down and spirals (ticks $t=201,369$ ). Parameters same as Fig. 1, except for a higher decay rate $\delta=0.15 \%$. (Video at http://tinyurl.com/qcofya 4 .)

Among the various parameters of the model, we chose to explore two in particular: the $M$-decay rate $\delta$ (at which $M$ 's transform into $W^{\prime}$ s) and the $C$-saturation level $c$ (beyond which the synthesis of $C$ 's is inhibited). The other main parameters were fixed, with following values: curvature angle $\alpha=8^{\circ}, C$-saturation radius $r=10, M$-vibration width $\varepsilon=0.05$. The outcome of simulation was evaluated differently in the two scenarios studied here: for maintenance autopoiesis, we measured the "lifespan" $\tau$ of viable cells and for emergence autopoiesis, we counted the closed cells together with the number and size of holes in their membrane. A summary of the setup follows. Detailed results are presented in the next section.

Maintenance autopoiesis, taken separately from emergence autopoiesis, refers to the survival of an existing system starting from a fully closed membrane, with no hole of any size (Fig. 4). Our initialization used a discretized circle or radius 10 (composed of $80 \mathrm{M}$ 's) located in the center of a world that was filled with $S$ particles only. The results varied from very short to very long lifespans. We measured these durations using the topological criterion based on a continally updated list of holes. Given the maximum hole size $\lambda$, the lifespan $\tau$ was set to the first tick when either the membrane was broken (the number of larger holes reached 2) or the cell started forming a spiral (the hub-and-spokes test was positive).

Emergence autopoiesis, on the other hand, is what we expect to happen when starting from a single $M$ particle. Under the right conditions, an $M$ catalyzes the reaction of $S$ particles on its "back", producing $C$ 's that later transform into new $M$ 's and so on. This creates a growing chain that should eventually close and form an autopoietic system. As in the maintenance case, the decision as to whether the simulation produced a stable system depended on an assessment of the membrane topology. In the emergence case, we used the same threshold value $\lambda=1.5$ but computed the number of closed cells only. We also screened out spiraling membranes. 


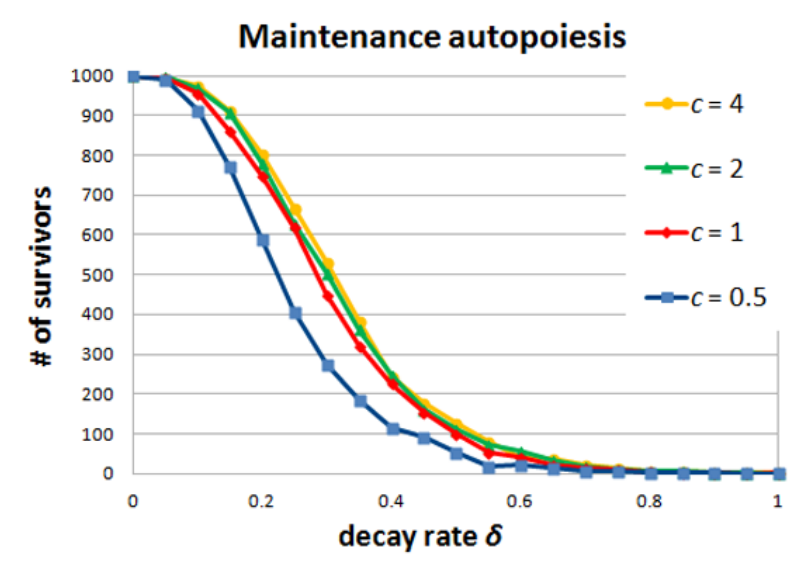

(a)
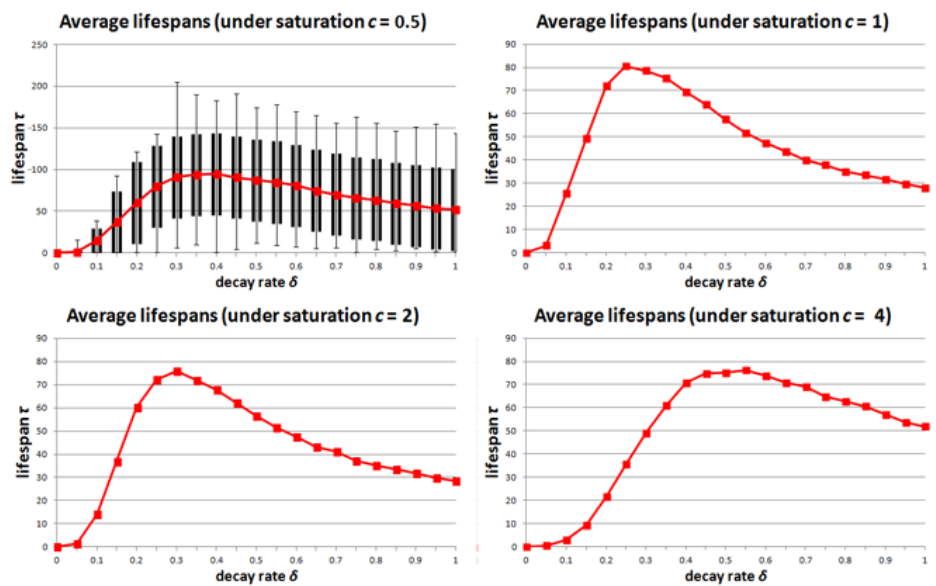

(b)

Figure 5: Statistical analysis of maintenance autopoiesis. (a) Number of surviving cells at $t_{N}=3000$ as a function of $M$-decay rate $\delta$ under four different $C$-saturation levels $c$. A cell is a "survivor" if its membrane contains at most one hole larger than 1.5 . Starting from 1000 cells, the half-population survival level 500 was reached for $\delta$ between $0.22 \%$ and $0.32 \%$. (b) Average lifespans $\tau$ of the non-survivor cells as a function of $\delta$ under the same four values of $c$. Top-left: boxplot showing the standard deviation and extremes of each distribution (see Fig. 6). Compared to survivors, for which $\tau \geq 3000$, non-survivors were wiped out relatively quickly, from immediately $(\tau=0)$ to about $\tau=200$. More importantly, the lifespan reached a peak for optimal values of $\delta$, between $0.25 \%$ and $0.55 \%$ depending on $c$, implying that some decay helped the cells in their regeneration process. Other parameters were the same as Fig. 1 .

\section{Results}

\section{Maintenance autopoiesis}

A statistical analysis of the model was performed on 1000 runs for each set of parameters. Each successful run lasted until tick $t_{N}=3000$. The $M$-decay rate $\delta$ was varied from $0 \%$ to $1 \%$ by increments of $0.05 \%$, and four different values of $C$-saturation level were tried: $c=0.5,1,2$, and 4 (Fig. 5). First, they confirmed that our model supported the "maintenance" form of autopoiesis, as a significant number of runs ended with closed or viable cells-which are referred to as "survivors" in this case. Although no upper limit was put on the size of the only allowed hole larger than $\lambda$ (for the cell to be at least viable), empirically we never observed holes larger than $h=4.5$, and such extremes occurred rarely. Thus there was no massive "unknitting" of the membrane.

The most striking observation is that the number of survivors started falling sharply for $\delta$ values as small as $0.1 \%$ and almost vanished at $0.7 \%$ (Fig. 5a). The half mark of 500 cells, roughly corresponding to the inflexion point of the curve, was reached by an average $\delta$ comprised between $0.22 \%$ (for $c=0.5$ ) and $0.32 \%$ (for $c=4$ ). This is intuitively reasonable, as the system cannot be autopoietic with too much decay or too little new material, and an increase in the former should be compensated by an increase in the latter to maintain the cell membrane.

A better insight into the behavior of the model could be gained by analyzing the average lifespan $\tau$ of non-survivor cells (Figs. 5b-6). Compared to survivors, which by definition reached at least $\tau=3000$, non-survivors died early in the simulation, generally before $\tau=200$. It means that autopoietic cells that have lasted beyond a certain time limit can expect to maintain themselves virtually forever. More interestingly, we found that, among the non-survivors, $\tau$ did not monotonously decrease with $\delta$, as it could be expected on first thought (i.e. the more decay, the earlier the membrane shoud collapse). Instead, $\tau$ first increased to a maximum comprised between 75 and 95 units of time (depending on $c$ ) for the lower range of $\delta$ values, then decreased again but more slowly. The maximum point was reached for an optimal $\delta$ between $0.25 \%$ and $0.55 \%$, a range similar to the inflexion point of the survivors' curve.

\section{Distribution of lifespans}

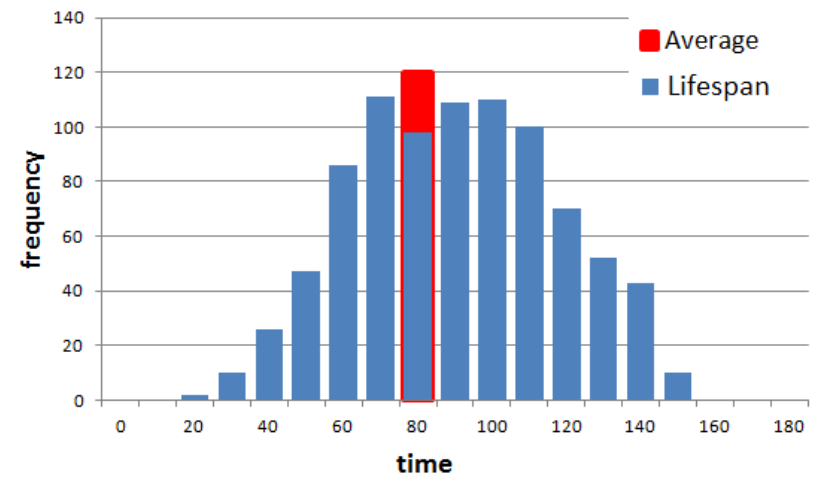

Figure 6: Distribution of lifespans $\tau$. This histogram was obtained from 1000 runs under a $C$-saturation level $c=4$ and an M-decay rate $\delta=0.5$. It would correspond to a boxplot placed in the middle point of the bottomright curve of Fig. $5 \mathrm{~b}$ (average $\tau$ of 75 ; bins are labeled here by their upper bound). As this batch produced 126 survivor cells, only 874 of 1000 were used to compute this histogram. Other parameters same as Fig. 1.

This seemed to indicate that some disruption of the membrane was necessary for the cells to express their autopoietic abilities. One explanation could be that when the membrane is initially disrupted through the decay of a few $M$ particles 

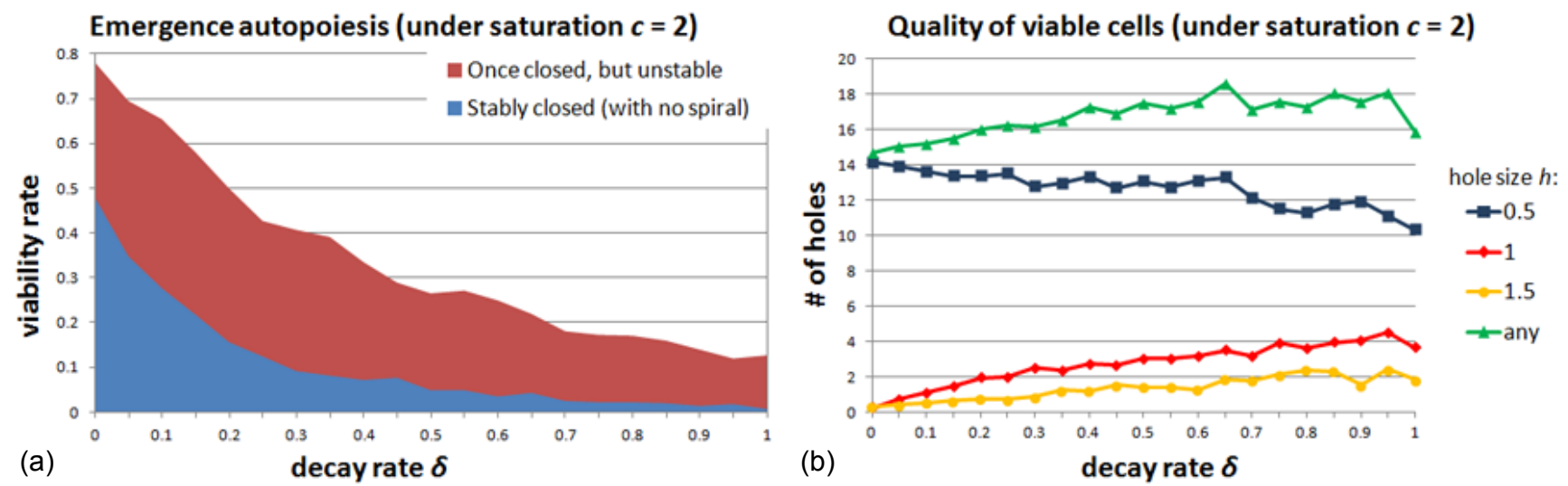

Figure 7: Statistical analysis of emergence autopoiesis. (a) Total number of "closed" newborn cells (as a fraction of the initial population of 1000). Blue area: cells that are still closed at the end of the simulation $\left(t_{N}=3000\right)$; red area: cells that reached a closed state but were unstable and did not survive. (b) Distribution of holes in the membrane of stable cells, plotted according to their size $h$. Three categories were defined: $0.5 \leq h<1$ ("category- 0.5 " holes), $1 \leq h<1.5$ ("category-1" holes), and $1.5 \leq h$ ("category-1.5" holes). Green curve: total number of holes (sum of the other three curves).

becoming $W$ 's, which drift away and create holes, the repair process often has a tendency to generate a pathological spiral configuration-but, precisely, this spiral is less likely to grow if it is itself disrupted by a moderate level of $M$-decay. In short, $\delta$ would need to be sufficiently high to destabilize spurious (and ultimately lethal) repair structures, but not as high as to riddle the membrane with too many holes that cannot be repaired quickly enough. This is a form of "creative destruction" that is reminiscent of cancerous cells.

\section{Emergence autopoiesis}

Next, we investigated the self-creation of well-formed autopoietic systems from a single monomer $M$, based on the same dynamics and parameters as maintenance autopoiesis. As before, results were collected from 1000 runs over a total duration of 3000 time units, and by varying $\delta$ from $0 \%$ to $1 \%$. The saturation level $c$ was set to a constant 2 (Fig. 7). They showed that our model also supported the "emergence" form of autopoiesis. Here, we only counted the number of cells capable of building a closed membrane, i.e. with no hole larger than $\lambda$. We distinguished between cells that were still stably closed at $t_{N}=3000$ and cells that were once closed but unstable and did not survive until the end of the simulation (Fig. 7a). Overall, the number of stable newborn cells was relatively low, starting at less than 500 (half the initial population) and dropping under 50 past $\delta=0.5 \%$. The total number of newborn cells, stable and unstable, was more significant, going from 800 to 250 within the same range.

In this emergence scenario, however, there was no "creative destruction" tendency: starting from $0 \%$, a moderate increase in $\delta$ did not produce more stable cells. This is possibly due to the fact that, unlike self-perpetuation, self-creation does not involve the repeated outgrowth of small spiral "barbs" on top of a circular membrane (defects which then need to be erased by noise), but produces a more consistent development of a longer structure-ending up either in a circle or a spiral. Moreover, the range of $\delta$ values favorable to the self-creation of a closed membrane from scratch might not overlap with the range of $\delta$ values that help an existing membrane regenerate itself. Therefore, when starting from a single $M$, and once $\delta$ has been set, it seems that the system can only bifurcate between a closed circle state and an open spiral state, but not transition from the latter to the former. A more complete chart of the system's "phase space" would be needed to confirm these hypotheses.

We also assessed the topology of stable cells by analyzing the distribution of holes in their membrane. Fig. $7 \mathrm{~b}$ plots the number of holes whose size falls into three categories delimited by $h=0.5,1$, and 1.5 , together with the total number of holes (of any size). While the number of category-1 and category-1.5 holes increased with the decay rate $\delta$, the number of category- 0.5 holes decreased. This could be an artifact of the simulation caused by the "receptor arc" of angle $\alpha$ limiting the conversion of C's into $M$ 's and making it more difficult for smaller holes to be repaired. Higher decay rates limit the occurrence of such cases but, naturally, add larger holes, too.

\section{Discussion}

We have proposed a minimal model of cell autopoiesis in continuous 2D space inspired by artificial chemistry. It contains three molecular types, a substrate, "components", and waste particles, which freely move in the environment, collide and react under specific conditions. Molecules of a fourth type, the monomers, bind to each other and occupy crystallike lattice positions, around which they vibrate, forming the cell's membrane. Results show that, under certain sets of parameters, our model is able to reproduce not only the traditional "maintenance" form of autopoiesis, but also build a new cell from scratch, displaying "emergence" autopoiesis.

Whereas other autopoietic models have been based on discrete cellular automata of various types (Varela et al., 1974; Zeleny, 1977; Breyer et al., 1998; McMullin and Groß, 2001; Sirmai, 2013) or autocatalytic sets of binary strings (Banzhaf, 1994), we have implemented a somewhat more realistic "dynamic billiard" agent-based simulation, similar to the molecular models used in microcanonical statistical me- 
chanics or in artificial chemistry (Ono and Ikegami, 2001: Chap. 3), and from which we could collect meaningful quantitative results. Most of the simplifications we adopted were intended to reduce the computational cost, in particular relying on a grid approximation to detect collisions faster. One especially contrived part of the model, however, is the absence of movement of the bound monomers (beside vibration), and the consequent rigidity of the membrane, which may not deform or drift once pegged to the underlying lattice partition of space. As for the curvature angle and receptor arc presiding over membrane polymerization (Fig. 2b), they can be interpreted as an abstract reflection of the internal geometric or "conformational" properties of monomer molecules.

The fact that a cell in our model can both maintain and create its own organization from a chaotic environment is a contribution toward uniting the three main perspectives and modeling approaches to life: origins (Rasmussen et al., 2004), autopoiesis, and self-replication (Langton, 1984; Sayama, 2000). The data obtained from our model further suggests that there is an optimal decay rate for autopoietic systems. Too much destruction of the membrane breaks the system down, while too little allows inevitable imperfections triggered by the environment's molecular randomness to thrive and take over the cell. This observation could be relevant to the study of oncological perturbations, or the early chemical environments favorable to the emergence of biotic forms.

In conclusion, the experiments presented here could provide another foundation on which to build more elaborate and realistic autopoietic models in continuous space. While we have already started working on an extension to a 3D world, other improvements should also focus on refinining the molecular mechanisms (collisions, geometry-based interactions and reactions; Fernández et al. 2012), and inserting some genetic information, implicitly or explicitly, to let the system evolve toward self-replication. The "emergent autopoiesis" side of our model also provides another illustration of the morphogenetic engineering viewpoint on self-organized complex systems (Doursat et al., 2012, 2013), for which the onset of order can be much more than random, "texture"-like patterns (stripes, spots, waves) and exhibit strong architectural features, too (nonhomogeneity, reproducibility, programmability). These morphogenetic capabilities have spontaneously evolved in nature over millions of years and could now be artificially accelerated and put to practical applications by human inventiveness. Swarm chemistry (Sayama, 2009), the growth of nontrivial shapes from mixed particle species, and synthetic biology, the design and control of "bioware" systems, are such examples at the molecular and cellular scale.

\section{References}

Banzhaf, W. (1994). Self-organization in a system of binary strings. Proceedings of Artificial Life IV, pp. 109-118.

Beer, R.D. (2004). Autopoiesis and cognition in the game of life, Artificial Life, 10:309-326.

Bourgine, P. and Stewart, J. (2004). Autopoiesis and Cognition, Artificial Life, 10(3):327-345.

Breyer J., Ackermann J. and McCaskill J. (1998). Evolving reactiondiffusion ecosystems with self-assembling structures in thin films, Artificial Life, 4:25:40.

Doursat, R., Sayama, H. \& Michel, O., eds. (2012). Morphogenetic Engineering: Toward Programmable Complex Systems. "Understanding Complex Systems" Series, Springer-Verlag.
Doursat, R., Sayama, H. and Michel, O. (2013). A review of morphogenetic engineering. "Frontiers of Natural Computing" (FNC 2012) Special Issue. Lones, M., Tyrrell, A., Stepney, S. \& Caves, L., eds. Natural Computing 12(2): 517-535.

Fernández, J. D., Doursat, R. \& Vico, F. J. (2012) The evolution of controller-free molecular motors from spatial constraints. Proc. of 5th Spatial Computing Workshop (SCW 2012), in 11th Int'l Conf. on Autonomous Agents and Multiagent Systems (AAMAS 2012), vol. W21, June 4-8, 2012, Valencia, Spain: pp. 19-24. IFAMAAS.

Fleischaker, G. R. editor (1992). Autopoiesis - A Debate: Controversy over Physical, Biological and Social Systems. International Journal of General Systems, Volume 21.

Hutton, T. J. (2007). Evolvable Self-Reproducing Cells in a twodimensional artificial chemistry, Artificial Life, 13(1):11-30.

Langton, C. G. 1984. Self-reproduction in cellular automata. Physica D 10:135-144.

Luhman, N. (1986). The Autopoiesis of Social Systems, Sociocybernetic paradoxes, pp. 172-192.

Maturana, H. R. and Varela, F. J. (1973). Autopoiesis: The Organization of the Living, in Autopoiesis and Cognition: The Realization of the Living (Maturana and Varela 1980), pp. 59-138. Dated 1973. Firs published 1972 in Chile under the title De Maquinas y Seres Vivos, Editorial Universitaria S.A.

Maturana, H. R. and Varela, F. J. (1980), Autopoiesis and Cognition: The Realization of the Living, Vol. 42 of Boston Studies in the Philosophy of Science, D. Reidel Publishing Company, Dordrecht, Holland. With a preface to 'Autopoiesis' by Stafford Beer. Series editors: Robert S. Cohen and Marx W. Wartofsky.

McMullin, B. (2004). 30 Years of Computational Autopoiesis: A Review, ALife, 10(3): 277-295.

McMullin, B. and Groß, D. (2001). Towards the implementation of evolving autopoietic artificial agents, in Advances in Artificial Life, Procedings of the $6^{\text {th }}$ European Conference on Artificial Life (ECAL2001), Kelemen, J. and Sosík, P. editors, pp. 440-443.

McMullin, B. and Varela, F. J. (1997). Rediscovering Computational Autopoiesis, ECAL-97 Presentation

Ono, N. and Ikegami, T. (1999). Model of self-replicating cell capable of self-maintenance, in Procedings of the $5^{\text {th }}$ European Conference on Artificial Life (ECAL1999), Floreano, D., Nicoud, J.-D. and Mondada, F. editors, pp. 399-406.

Ono, N. and Ikegami, T. (2000). Self-maintenance and self-reproduction in an abstract cell model, J. of Theoretical Biology, 206:243-253.

Ono, N. and Ikegami, T. (2001). Artificial chemistry: Computational studies on the emergence of self-reproducing units, in Advances in Artificial Life, Proc. of the $6^{\text {th }}$ European Conference on Artificial Life (ECAL2001), Kelemen, J. and Sosík, P. editors, pp. 186-195.

Pelaez, N. (2009). Autopoiesis Model, EECS 372/472 Course at Northwestern University. http://ccl.northwestern.edu/courses/mam2009/ student work/ Autopoiesis.html.

Rasmussen, S., Chen, L., Deamer, D., Krakauer, D. C., Packard, N. H., Stadler, P. F., \& Bedau, M. A. (2004). Transitions from nonliving to living matter. Science, 303(5660), 963-965.

Sayama, H. (2000). Self-replicating worms that increase structural complexity through gene transmission, Artificial Life VII: Proc. of the $7^{\text {th }}$ Int'l Conference on Artificial Life, M. A. Bedau, J. S. McCaskill, N. H. Packard and S. Rasmussen, eds., pp.21-30, MIT Press.

Sayama, H. (2009). Swarm Chemistry, Artificial Life, 15(1):105-114.

Sirmai, J. (2011). A schematic Representation of Autopoiesis Using a new kind of Discrete Spatial Automaton, $11^{\text {th }}$ European Conference on Artificial Life (ECAL2011)

Sirmai, J. (2013). Autopoiesis facilitates Self-Reproduction, $12^{\text {th }}$ European Conference on Artificial Life (ECAL2013)

Swenson, R. (1992). Galileo, Babel and Autopoiesis (It's turtles all the way down), International Journal of General Systems, 21:207-228.

Varela, F. J., Maturana, H. R. and Uribe, R. (1974). Autopoiesis: The Organization of Living Systems, its Characterization and a Model, BioSystems, 5:187-196.

Wilensky, U. (1999). NetLogo, ccl.northwestern.edu/netlogo, Center for Connected Learning and Computer-Based Modeling, Northwestern University, Evanston, IL.

Zeleny, M. (1977). Self-Organization of Living Systems: A formal Model of Autopoiesis, International Journal of General Systems, 4:13-28. 